\title{
Use of liquid-gas ejector in liquefied natural gas (LNG) sampling system
}

\author{
Y.V. Martynenko ${ }^{1}$, V.I. Bolobov ${ }^{1}$, V.A. Voronov ${ }^{2}$ \\ ${ }^{1}$ Saint Petersburg Mining University, Department of Transport and storage of oil and gas, Oil and gas \\ faculty, Russia \\ ${ }^{2}$ ITMO University, Saint Petersburg, Faculty of biotechnologies and low-temperature systems, Russia
}

\begin{abstract}
The article considers the modernization of the periodic sampling system of liquefied natural gas (LNG)by introducing a liquid-gas ejector (LGE) as an alternative to a gas compressor. The unique properties of liquefied natural gas allow the fuel energy to be directed to the ejector without any externalenergy input. Besides, the advantage of this method is that it prevents changes in the original chemical composition of the sample due to the liquid-gas ejector, which does not require lubricating oils. Also, the system reduces the volume of the regasified sample and eliminates the possibility of ejector failure.
\end{abstract}

\section{Introduction}

The large increase in the production of liquefied natural gas has led to such significant globalization of the gas market that the rapidly expanding accompanying infrastructure is no longer able to keep up with [1]. B.S.Rachevsky in his book "Liquefied hydrocarbon gases" gives experts "forecasts that the trend of accelerated growth in gas production and use in the 21 stcentury will intensify, and the gas industry will become a leader in the structural improvement of the global energy balance [2]. Against this background, domestic developments and investments in the development of liquefied natural gas in Russia are particularly relevant. According to the latest data presented in BP's annual statistical report, the share of explored natural gas reserves in Russia amounts to 32.3 trillion cubic meters, which is $17.3 \%$ of all world reserves. At present, almost 643 billion cubic meters or $18.03 \%$ of the total yearly gas production are produced. Russia is the leader in the amount of natural gas reserves, so it is economically feasible to develop technologies for their extraction and use [3].

Even though most of the domestic regulatory framework for the production and use of liquefied natural gas (LNG) is under development, GOST R 56719-2015 came into force in 2017[5]. According to this GOST, sampling operations should be performed during the entire period of carrying out the following types of work [6]:

- Operation of the natural gas liquefaction plant

- Supply of LNG to a container or storage facility

- $\quad$ LNG shipments to vehicles

- LNG supply to regasification plants

- Other processes for transferring LNG to consumers. 
There is a continuous and periodic sampling of LNG. Continuous sampling from the LNG flow is performed permanently with subsequent regasification and accumulation in the tank to obtain an integral sample. Periodic sampling is the calculation of spot samples (at least three) from an LNG or regasified liquefied natural gas stream on a uniform time basis or in equal volumes.

To analyze current issues of development of the liquefied natural gas market, development of regulatory documentation in this area in the Russian Federation, as well as existing technological schemes of hydrocarbon transportation $\mathrm{s}$, the authors were guided by sources $[7,8]$ in order to form technical solutions for improving the LNG sampling system.

\section{Ejector system for sampling}

In practice, a large number of sampling schemes are used.

When reviewing of the patent base, we considered a method of gas sampling, which involves moving gas from the controlled environment into the gas discharge line under the influence of rarefaction [9]. It is created by a jet of air directed through the ejector, formed under the impact of the pressure difference in the environment and the flow of the controlled medium. The disadvantage of this method is the removal of gas after the analysis by introducing it into a directional air stream, which leads to mixing of streams and changes in the component composition in the controlled environment. In addition, the system provides for pumping fuel only ate pressure below atmospheric pressure, because the known method is based on using air from the environment as the working flow, so it can be used only if the pressure in the flow of the controlled environment is below the air pressure.

The continuous LNG sampling method with a compressor and a hydraulically sealed gas cylinder includes a compressor in the sampling sheme, which serves to supply the gas sample to the sampler (GOST R 56719-2015). Its disadvantage is the possibility of changing the component composition of the supplied sample due to the ingress of lubricating oils from the gas compressor, as well as additional capital and energy costs when completing and operating the system, respectively. In addition, the use of a compressor leads to a decrease in the reliability of the system of continuous selection process as a whole and the necessity of additional equipment maintenance.

Currently, the method of periodic sampling of LNG with constant pressure samplers is most widely used for LNG sampling at storage facilities in the Russian Federation [5]. The method consists of sampling LNG, regasifying it maintaining the required pressure, and transporting the sample for chemical analysis; the sample gas is pumped into the sampler using a gas compressor (Figure 1). However, the ingress of gas compressor lubricants into the sample may change its component composition. Besides, such a system requires additional capital and energy costs for its configuration and operation. 


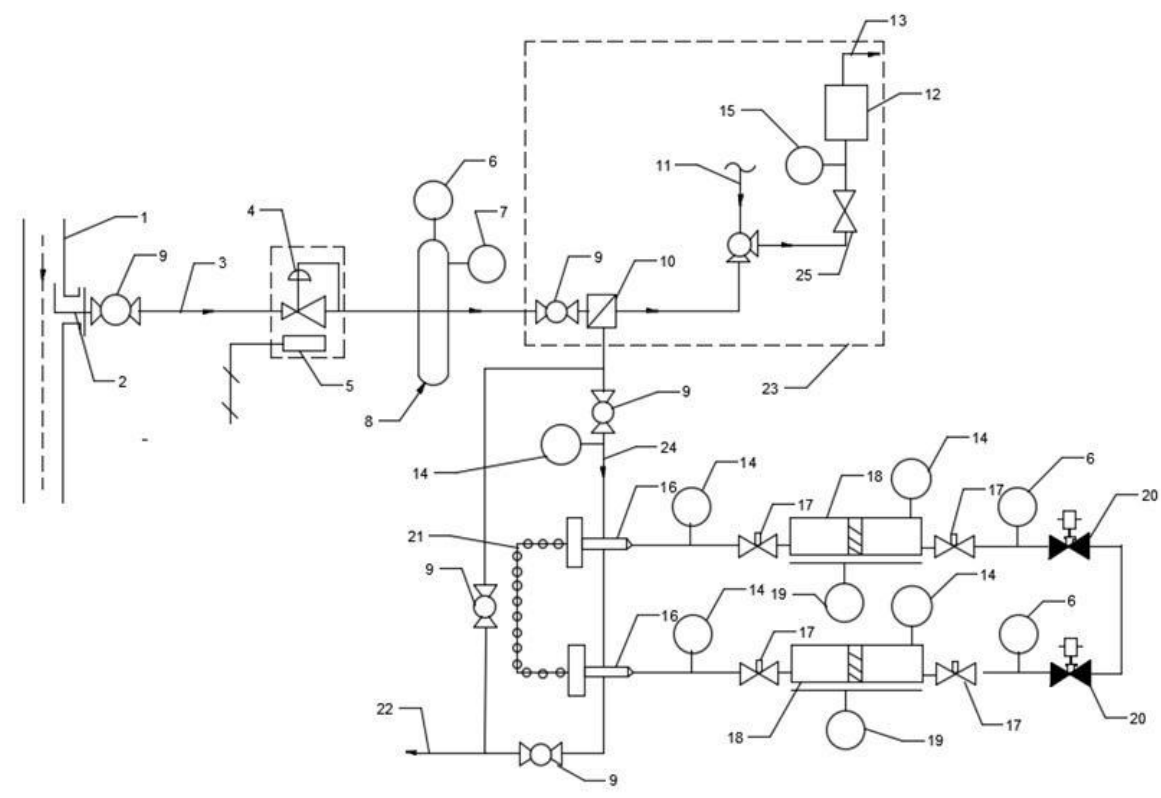

Fig. 1. Periodic sampling scheme for LNG with gas compressors: 1 - LNG pipeline, 2 - sampling probe, 3 - sampling line, 4 - vaporizer, 5 - heater, 6,14 - pressure gauge, 7 - thermometer, 8 accumulator, 9 - valve, 10 - sample filter, 11 - calibration gas, 12 - gas chromatograph, 13 discharge, 15 - flow meter, 16 - gas compressor, 17 - needle valve, 18 - constant pressure sampler, 19 - level sensor, 20 - solenoid valve, 21 - air supply by blowers (high-pressure flow), 22 -gas pipeline - offsetting for technological needs, 23 - chromatograph, 24 - the pipeline of regasified LNG, 25 - gate valve.

The sampling technique used in practice is as follows (Figure 1): LNG going through pipeline 1 is sampled by sampler 2 and fed through sampling line 3 to vaporizer 4 for regasification. Then one part of the sample is sent to chromatograph 23, and the other part goes through pipeline 24 to gas compressor 16, which pumps the sample into a constantpressure sampler 18 already in the liquefied state. Accumulator 8 is used to smooth out pressure pulsations of the regasified sample. The flow rate of LNG supplied to the vaporizer is set by valve 9 .

This article proposes a method for improving this method, characterized by the fact that the regasified liquefied natural gas, after passing the gas chromatograph, is pumped into the sampler using an ejector that serves as an alternative to the compressor (Figure 2).

In accordance with GOST R 56719-2015, the absolute pressure of regasified LNG after the vaporizer must be maintained in the range from 0.25 to $1.0 \mathrm{MPa}$ using pressure regulator 26 included in the modernized scheme. The required pressure in the LNG pipeline 1 is $0.25 \mathrm{MPa}$. Having connected ejector 16 with sampling line 21 to LNG pipeline 1 , the liquid phase should enter the ejector, while being a low-pressure flow, and the regasified LNG is a high- pressure one, provided that regulator 26 is set to a pressure exceeding the lower limit of $0.25 \mathrm{MPa}$. 


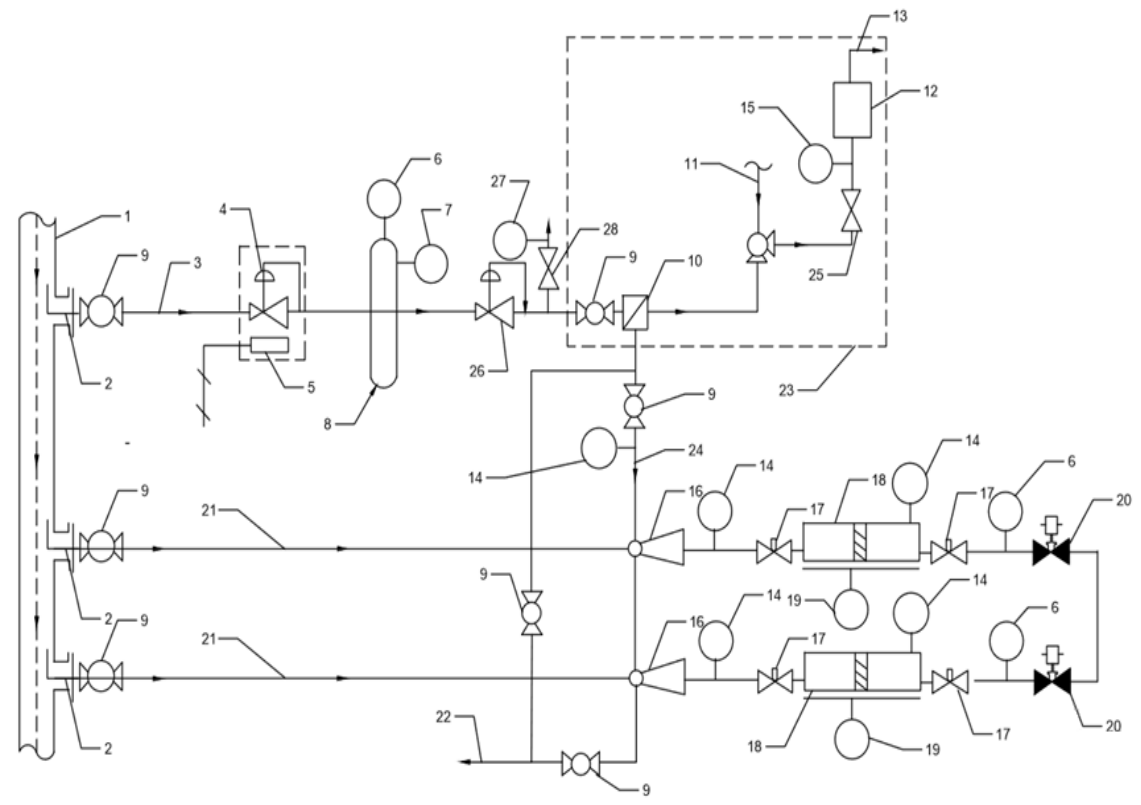

Fig. 2.Modernized periodic sampling scheme for LNG with ejectors: 1 - LNG pipeline, 2 - sampling probe, 3 - sampling line, 4 - vaporizer, 5 - heater, 6,14,27 - pressure gauge, 7 - thermometer, 8 accumulator, 9 - valve, 10 - sample filter, 11 - calibration gas, 12 - gas chromatograph, 13 discharge, 15 - flow meter, 16 - liquid-gas ejector, 17 - needle valve, 18 - constant pressure sampler, 19 - level sensor, 20 - solenoid valve, 21 - LNG pipeline for sampling, 22 -gas pipeline - offsetting for technological needs, 23 - chromatograph, 24 - the pipeline of regasified LNG, 25,28 - gate valves, 26 -pressure controller.

Since a stable high-pressure flow must be provided due to the settings of the pressure regulator (Figure 2), the sample is injected into the sampler due to the created discharge area in the ejector mixing chamber. The unique properties of liquefied natural gas allow directing the fuel energy to the ejector without energy input from outside.

Thus, the proposed periodic sampling method of liquefied natural gas must be implemented according to the algorithm shown in Figure 3. 


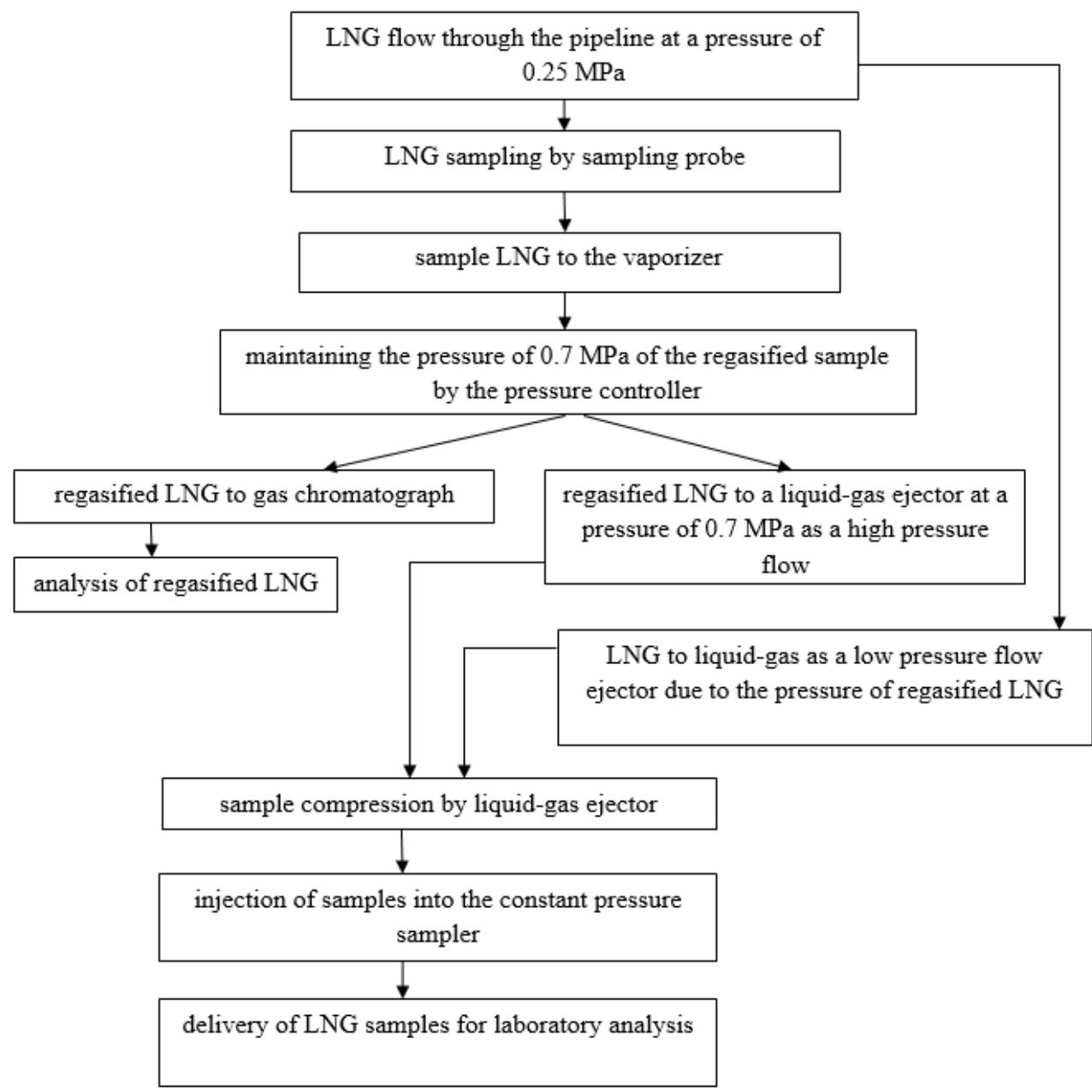

Fig. 3. Algorithm for periodic LNG sampling.

The advantage of this method is that the system of periodic sampling of LNG allows to prevent changes in the initial chemical composition of the sample of liquefied natural gas since the operation of a liquid-gas ejector does not require lubricating oils. Also, it reduces the volume of the sample undergoing regasification by selecting its part in the form of LNG with a liquid-gas ejector. Besides, the use of an alternative to the compressor device, such as a liquid-gas ejector, does not require external energy input and eliminates the possibility of failure of the device, and reduces capital costs for the equipment of such an LNG sampling system.

\section{Theoretical calculation of liquid-gas ejector characteristics and choice of aerodynamic scheme for sampling}

The following parameters were determined for the calculations:

- LNG mixture composition ( $\mathrm{CH}_{4}=95,5 \% ; \mathrm{C}_{2} \mathrm{H}_{6}=2,3 \% ; N_{2}=1,7 \%$ etc.) [15]

- $\mathrm{LNG}$ compression ratio at the ejector inlet $z=0,0396$ 
- LNG temperature at the ejector inlet $T_{f}=120 \mathrm{~K}$

- Absolute LNG pressure at the ejector inlet $P_{f}=0,25 \mathrm{MPa}$

- Vapor pressure at the ejector inlet (after pressure increase) $P_{g}=0,7 \mathrm{MPa}$

- $\quad$ Fuel flow rate $Q_{f 0}=89 \mathrm{dm}^{3} / \min$

- $\quad$ Saturated vapor pressure $P_{s}=0,192 \mathrm{MPa}$.

Based on the data presented in GOST R 56851-2016, a mixture No. 2 simulating LNG was selected, according to which the calculated values of thermodynamic properties are presented. Depending on the set absolute pressure of LNG, regulated by GOST R 567192015, the parameters of the liquid phase of the fuel compression ratio and temperature are selected (GOST R 56719-2015). The value of the saturated vapor pressure is set according to GOST R 56021-2014 and in accordance with the fuel temperature equal to $120^{\circ} \mathrm{K}$ [16]. Since the absolute pressure of regasified LNG after the vaporizer must be maintained in the range from 0.25 to $1.0 \mathrm{MPa}$, the vapor pressure at the ejector inlet is chosen as the average value of $0.7 \mathrm{MPa}$. According to GOST R 56719-2015, the system is equipped with a constant pressure sampler with a volume from $0.5 \mathrm{dm}^{3}$ to $1 \mathrm{dm}^{3}$. The required volume is provided by the internal volume flow of LNG and the flow of pumped regasified LNG.

The calculation was made in accordance with the methodology presented in the monograph "Hydraulic drive jet compressor units" by K.G.Donec([3]. However, in his formulas, the author uses liquid as the working flow (high pressure) for a liquid-gas ejector. In the modernized scheme, on the contrary, the working flow is the flow of regasified LNG, since it is due to its energy that the mixture is pumped into the sampler. Therefore, the formulas were corrected to reflect these changes $[13,14]$.

The results of calculating the main parameters, taking into account the coefficients for the optimal regime, are presented in Table 1, where: $u_{\text {optm }}$ is the coefficient of ejection (inner coefficient of displacement rate); $Q_{g}$ is the flow rate of working gas flow; $\varphi_{\text {optm }}$ is the coefficient of pressure recovery; $\varepsilon$ is the gas compression ratio; $P_{m i x}$ is the mixture pressure on the way out of liquid-gas ejector; $N$ is the power, spent on compressing while the ejector is working, with accuracy up to the efficiency coefficient of the pump; $N_{\text {reduce }}$ is the reduced power spent on compressing, $\eta$ is the efficiency coefficient.

Table 1. The results of calculating the characteristics of the ejector at the optimal regime.

\begin{tabular}{|c|c|c|c|c|c|c|c|c|}
\hline $\begin{array}{c}\text { Type of } \\
\text { scheme }\end{array}$ & $u_{\text {optm }}$ & $Q_{g}, \mathrm{M}^{3} / u$ & $\varphi_{\text {optm }}$ & $\varepsilon$ & $P_{\text {mix }}, M P a$ & $\begin{array}{c}N, \\
M W\end{array}$ & $N_{\text {reduce }}$ & $\eta, \%$ \\
\hline 1 & 1.969 & 0.336 & 0.283 & 3.193 & 0.377 & 2.349 & 61.288 & 41.075 \\
\hline 2 & 2.610 & 0.253 & 0.185 & 2.439 & 0.333 & 1.772 & 46.244 & 36.823 \\
\hline 3 & 3.115 & 0.212 & 0.151 & 2.169 & 0.318 & 1.485 & 38.742 & 36.602 \\
\hline 4 & 4.190 & 0.158 & 0.107 & 1.827 & 0.298 & 1.104 & 28.804 & 36.431 \\
\hline
\end{tabular}

The choice of the aerodynamic scheme of the ejector depends on the most effective indicators such as efficiency, ejection coefficient, and reduced power. It is recommended to choose an aerodynamic scheme to provide the highest efficiency at the calculated value of the compression ratio. In a liquid-gas ejector, the highest efficiency is achieved if the process of exchanging the amount of movement between the active and passive flows is completed within the working chamber and pumped before entering the diffuser, which is most consistent with scheme №1 according to the calculation results. [12] The design parameters of this scheme under specified conditions allow to get an efficiency of $41 \%$, which is a relatively high indicator for a pumpless ejector. The maximum experimental 
value that scientists were able to obtain in the optimal mode for the considered types of aerodynamic schemes of liquid-gas ejector is an efficiency equal to $40 \%$. Due to the highpressure difference between the LNG flows and the regasified sample, as well as the relatively low ejection coefficient for the selected type of aerodynamic scheme №1, the theoretical calculations made possible to receive energy-efficient results. Also, we took into account the method of A.P. Erokhin when calculating parameters of the ejector [11].

To visualize the obtained results, the dependences of the mixture pressure at the ejector outlet, reduced power and efficiency coefficient on the ejection coefficient for the four considered aerodynamic schemes are constructed (Figure 4).

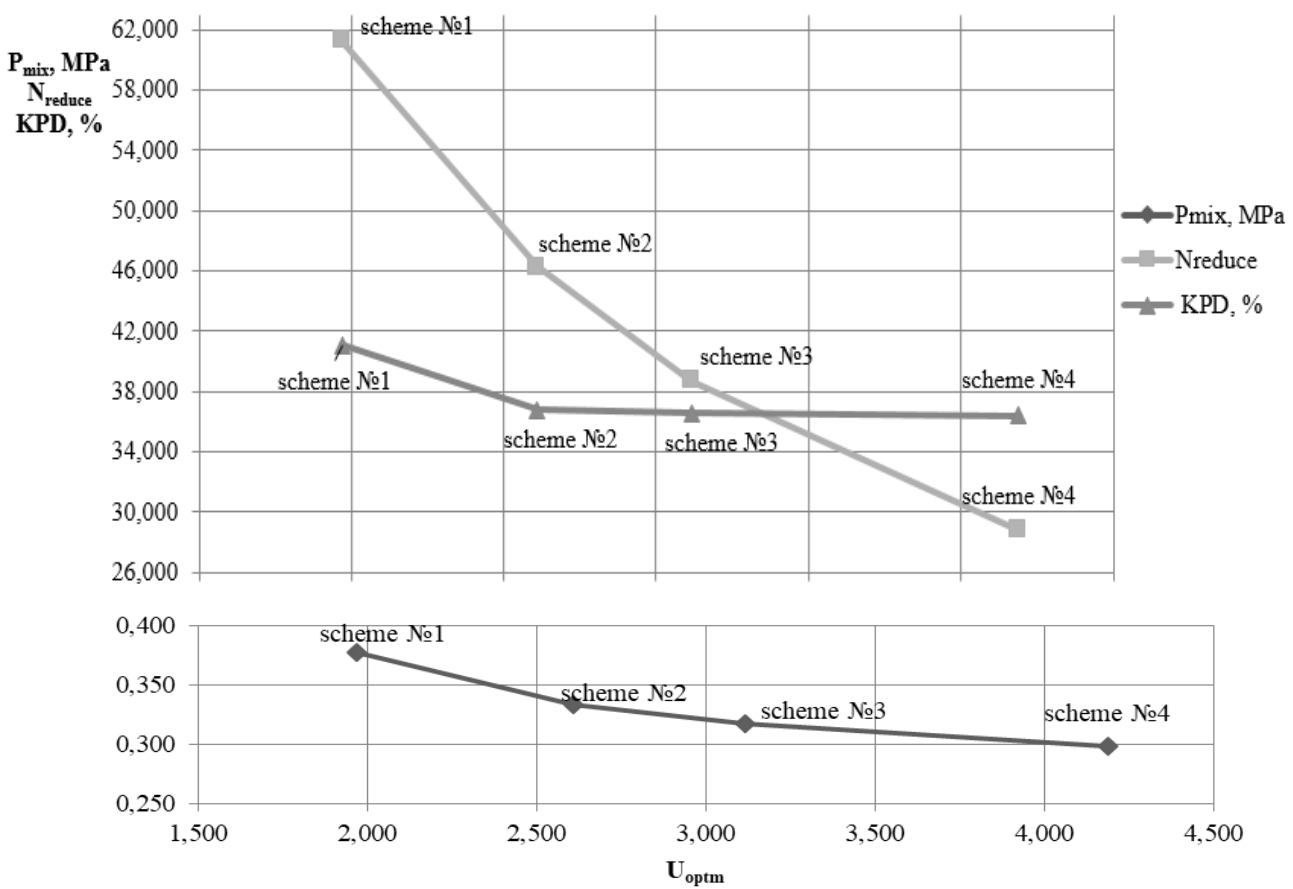

Fig. 4. Dependence diagram of the mixture pressure at the rejectoroutlet, the reduced power, and the efficiency coefficient on the ejection coefficient.

Thus, the aerodynamic scheme №1 is the most effective, since its geometric characteristics make it possible to obtain the highest values of efficiency, power, and pressure of the mixture.

According to the chosen scheme, the parameters of the ejector were calculated at the limiting and stalling mode of the liquid-gas ejector, and a pressure characteristic was constructed $P_{\text {mix }}=f\left(Q_{g}\right)$ (Figure 5). The calculation of the ejector operation at the limit and stall modes was also carried out according to the method of K.G.Donec, taking into account the coefficients presented by the author for the selected type of aerodynamic scheme of the ejector. By analogy, A.N. Drozdovand, Ya.A. Gorbyleva constructed the pressure characteristics of the ejector system in the article [10]. 


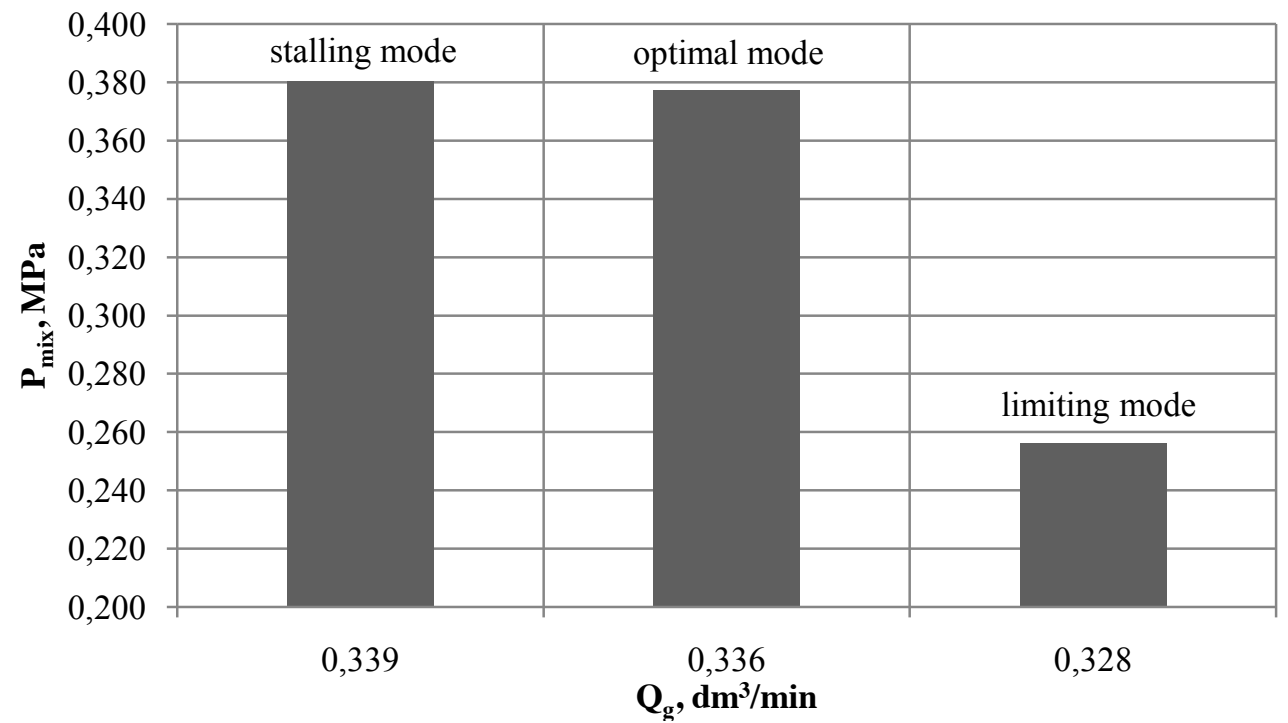

Fig. 5. Pressure characteristic for the aerodynamic scheme No.1 [11].

\section{Calculation of geometrical parameters}

To determine the geometric parameters of the selected ejector scheme, the nozzle diameter (equation 1) and the diameter of the working chamber are calculated (equation 2). The remaining dimensions of the flow section are selected according to Table 6 .

$$
d_{0}=\sqrt{\frac{4 Q_{g}}{\pi \cdot \mu_{n o z} \cdot i} \cdot \sqrt{\frac{\rho_{g}}{2\left(P_{g}-P_{f}\right)}}},
$$

Where $\mu_{n o z}$ is the coefficient to nozzle flow rate ( $\mu_{n o z}=0,79$ for scheme №1), $i_{\text {is }}$ the number of nozzles $(i=7)$.

$$
d_{w c}=d_{0} \cdot \sqrt{i \cdot m}
$$

Where $m$ is the coefficient for scheme $1(\mathrm{~m}=3,2)$.

The initial parameters were used to calculate the geometric dimensions of the flowing part of the liquid-gas ejector. The results are shown in Table 2.

Table 2. Geometrical dimensions of the flowing part of liquid-gas ejector

\begin{tabular}{|l|l|l|l|l|l|l|l|l|l|}
\hline $\begin{array}{l}d_{0} \\
, \mathrm{~m}\end{array}$ & $a, \mathrm{~m}$ & $\begin{array}{l}D_{0} \\
, \mathrm{~m}\end{array}$ & $\begin{array}{l}D_{1} \\
, \mathrm{~m}\end{array}$ & $\begin{array}{l}d_{2} \\
, \mathrm{~m}\end{array}$ & $\begin{array}{l}d_{w c} \\
, \mathrm{~m}\end{array}$ & $l_{\text {noz }, \mathrm{m}}$ & $l_{1}, \mathrm{~m}$ & $l_{2}, \mathrm{~m} *$ & $l_{\text {diff }}, \mathrm{m}^{*}$ \\
\hline 0.008 & 0.0024 & 0.011 & 0.025 & 0.034 & 0.038 & 0.1552 & 0.001 & 0.414 & 0.0575 \\
2 & 6 & 7 & 9 & 5 & 8 & & 4 & & \\
\hline
\end{tabular}

$$
l_{1}=\frac{d_{w c}-d_{2}}{2 t g \cdot \frac{\gamma_{1}}{2}} l_{d i f f}=\frac{2 \cdot d_{w c}-d_{2 y}}{2 t g \cdot \frac{\gamma_{d i f f}}{2}} .
$$

The calculated geometric values, depending on the diameters of the nozzle and the the working chamber, are not enough to build a three-dimensional model of the 
device, therefore, it is necessary to use a unified model to clarify the overall, center-to-center dimensions, as well as the length of the mixing chamber (Table 3):

Table 3.Overall and center-to-center dimensions of the liquid-gas ejector structure

\begin{tabular}{|c|c|c|c|c|c|c|}
\hline$D_{y 1}, \mathrm{~m}$ & $D_{y 2}, \mathrm{~m}$ & $D_{y 3}, \mathrm{~m}$ & $L_{1}, \mathrm{~m}$ & $L_{2}, \mathrm{~m}$ & $L_{3}, \mathrm{~m}$ & $l_{w c}, \mathrm{~m}$ \\
\hline 0.050 & 0.050 & 0.050 & 1.800 & 0.200 & 0.800 & 0.1969 \\
\hline
\end{tabular}

The calculated geometric values correspond to the constructive scheme of the flow part of the ejector, shown in Figure 6.

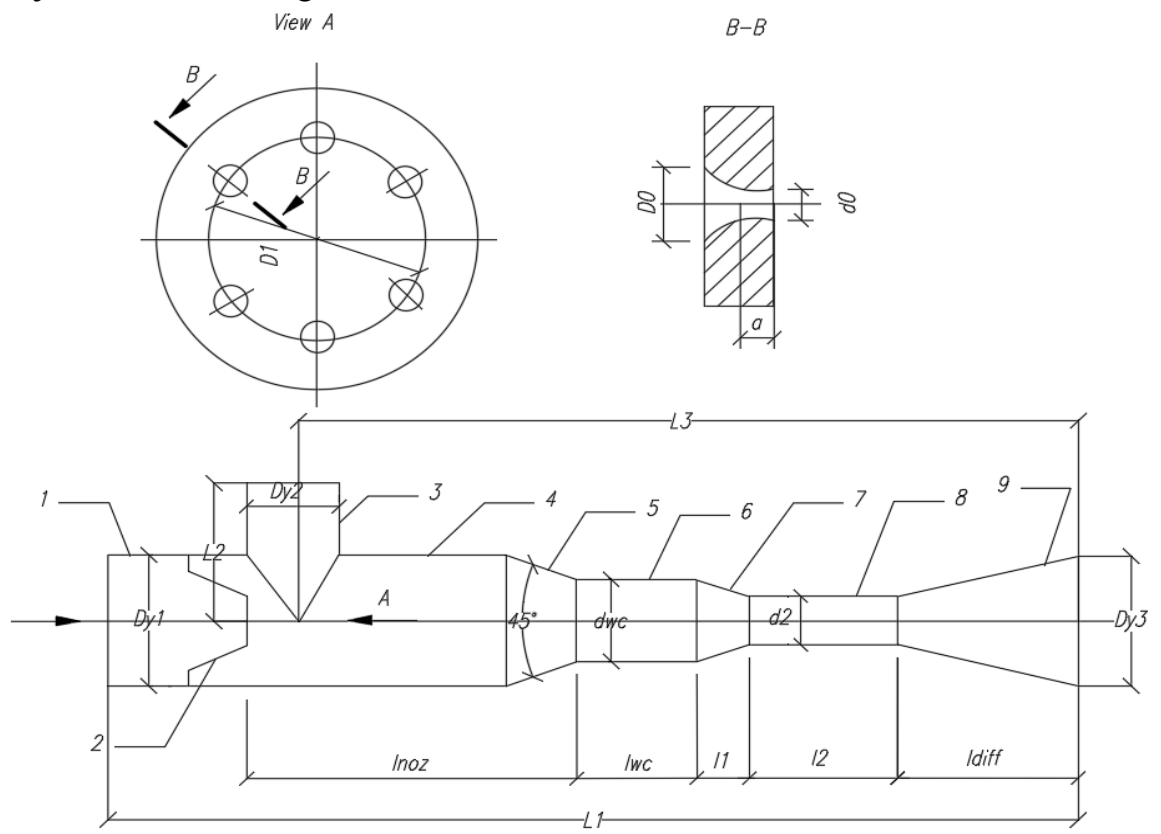

Fig. 6. Constructive diagram of the flow part of the ejector: 1 - nozzle in let working gas; 2 -nozzle; 3 - receiving pipe; 4 - thepre-chamber; 5 - confuser; 6 - mixing chamber; 7 -convergent phase; 8 cylindrical portion; 9 - diffuser area.

\section{Conclusion}

As an alternative to traditional methods of periodic sampling of LNG, it is proposed to use a modernized scheme with liquid-gas ejectors, in which regasified LNG, after passing a gas chromatograph, is pumped into the sampler using an ejector.

To justify the efficiency of using a liquid-gas ejector, the main indicators of its efficiency were calculated, according to which the most productive aerodynamic scheme was selected. For visual analysis of the obtained results, the dependences of the mixture pressure at the outlet of the ejector, the reduced power, and the efficiency coefficient on the ejection coefficient were constructed. In accordance with the selected scheme, the pressure characteristic of the device operating at optimal, stall, and limit modes was constructed. The geometric dimensions of the flow part of the ejector were calculated and its design scheme was presented. 


\section{References}

1. K.V. Remishevskaia, D.Yu. Zakharov, Yu.S. Gontar, Actual problems of effective and safe development of the Arctic oil and gas fields using the case of the Yamal peninsula. Electronic scientific journal oil and gas business5: 151-174 (2018).

2. B.S. Rachevsky, Liquefied hydrocarbon gases. (Moscow: "OIL and GAS", 2009).

3. K.G. Donec, Hydraulic drive jet compressor units. (Moscow: Nedra, 1990).

4. The official website of British Petroleum. Mode of access: https://www.bp.com. (Accessed 15.04.2020).

5. GOST R 56719-2015. Natural gas, liquefied. Sampling. (M.:Standartinform, 2016).

6. GOST R 55892-2013. Objects of low-tonnage production and consumption of liquefied natural gas. General technical requirements. (M.: Standartinform, 2014).

7. E.A. Lyubin, K.A. Zaynetdinov, D.Yu. Belov, Evaluation of the effectiveness of the combined membrane-ejector vapour recovery system. Petroleum Engineering 18(3): 69-79(2020).

8. M.N. Nazarova, E.A. Nazarova Modern approach to the normative legal base in the field of construction of large-capacity LNG plants. Youth energy for the oil and gas industry. Materials of the III International scientific and practical conference of young scientists: 304-307 (2018).

9. V.V. Nechaev, M.I. Saparov, S.A. Fadeev, Patent "Method and device for sampling gas" RU 2173841 (2001).

10. A.N. Drozdov, Ya.A. Gorbyleva, Improving the operation of pumping and ejector systems with changing associated petroleum gas flow rates. Notes of the Mining Institute 238: 415(2019).

11. A.P. Erokhin, Calculation of optimal parameters of the ejector. Notes of the Mining Institute1(65): 32 (1973).

12. N.In. Morozova, A.A. Korshak, Universal characteristics of liquid-gas ejectors. Oil and Gas business (electronic scientific journal)6: 368-383 (2013).

13. V.A. Voronov, Y.V. Martynenko, Engineering design of the ejector system for liquefied natural gas (LNG) vapor discharge. Topical Issues of Rational Use of Natural Resources 2019 - Litvinenko (Ed) (C) 2020 Taylor \& Francis Group, London: 107-113 (2019).

14. V.A. Voronov, Y.V. Martynenko, Technological solutions set to ensure the safety of storage of liquefied natural gas. Youth technical sessions proceedings, CRC Press/Balkema is an imprint of the Taylor \& Francis Group, an informa business: 842850 (2019).

15. GOST R 56851-2016 Liquefied natural gas. Thermodynamic properties calculation method. (M.:Standartinform, 2016).

16. GOST R 56021-2014. Combustible natural gas LPG. Fuel for internal combustion engines and power plants. Technical conditions. (M.:Standartinform, 2016). 